\title{
Considerations for the scaling-up of water splitting catalysts
}

\section{Kibsgaard, Jakob; Chorkendorff, Ib}

\section{Published in:}

Nature Energy

Link to article, DOI:

10.1038/s41560-019-0407-1

Publication date:

2019

Document Version

Peer reviewed version

Link back to DTU Orbit

Citation (APA):

Kibsgaard, J., \& Chorkendorff, I. (2019). Considerations for the scaling-up of water splitting catalysts. Nature Energy, 4(6), 430-433. https://doi.org/10.1038/s41560-019-0407-1

\section{General rights}

Copyright and moral rights for the publications made accessible in the public portal are retained by the authors and/or other copyright owners and it is a condition of accessing publications that users recognise and abide by the legal requirements associated with these rights.

- Users may download and print one copy of any publication from the public portal for the purpose of private study or research.

- You may not further distribute the material or use it for any profit-making activity or commercial gain

- You may freely distribute the URL identifying the publication in the public portal

If you believe that this document breaches copyright please contact us providing details, and we will remove access to the work immediately and investigate your claim 


\title{
Considerations for the scaling-up of water splitting catalysts
}

\author{
Jakob Kibsgaard and Ib Chorkendorff \\ SurfCat, Department of Physics, Technical University of Denmark \\ 2800-DK Kgs. Lyngby, Denmark \\ JK: jkib@fysik.dtu.dk and IC: ibchork@fysik.dtu.dk
}

\begin{abstract}
A wealth of candidates are being investigated to improve the catalysts found in acidic and alkaline electrolysers. However, attention should be focused on developing stable water oxidation catalysts with improved intrinsic activity - not only increased geometric activity - alongside bestpractice guidelines for data collection.
\end{abstract}

\section{Main}

A potential key storage solution for highly renewable power systems is the conversion of electrical energy into chemical energy in the form of fuels. It could also allow for a greater penetration of renewable energy in the chemical industry. ${ }^{1}$ The first and simplest step in this process could be to split water and generate hydrogen. There are two principal routes for this: in the near-term, electricity could be used to drive electrolyzers; in the longer term, the electrolysis process could be directly integrated with photovoltaics (PV) in a photoelectrochemical (PEC) device. ${ }^{2}$ Either way, to split water efficiently, both PV-electrolysis and PEC cells require highly performing catalysts for the two half reactions, the hydrogen evolution reaction (HER) and the oxygen evolution reaction (OER).

Water splitting can be done under either acidic or alkaline conditions. In acid, HER and OER have traditionally relied on platinum (Pt) and iridium (Ir), respectively, to catalyze the reactions. The availability of these precious metals motivates many efforts to find earth-abundant replacements. Yet in doing so, it is important to carefully consider how they are evaluated and compared.

\section{Impact of metal scarcity on scale-up}

The HER is one of the simplest electrochemical processes, involving only transfer of two electrons. Platinum is the archetypal catalyst but is also extremely scarce, mined in only three places in the world where it has been up-concentrated by geological events." Its exceptional HER activity (Figure 1) means that even very low platinum loadings at the cathode can provide sufficient activity to facilitate HER on the terawatt scale (the global energy demand reached 18 TW in $2016 .{ }^{4}$ If one is willing to accept a $50 \mathrm{mV}$ overpotential (the extra voltage required beyond the thermodynamic minimum to drive the reaction) at $10 \mathrm{~mA} \mathrm{~cm}^{-2}$ in a PEC device, it would require 54 tonne platinum - equivalent to $30 \%$ of annual production - to produce devices that generate hydrogen at a rate equivalent to $1 \mathrm{TW}$ of energy storage $\left(\mathrm{TW}_{\mathrm{H}_{2}}\right) \cdot{ }^{5}$ Even lower platinum loadings may be possible with the platinum dispersed as single atoms or small clusters. ${ }^{6}$ Similar arguments can be made for electrolyzers. to fabricate an electrolysis devices that generates $\mathrm{H} 2$ at a rate equivalent to 1 TW of energy storage 
Polymer electrolyte membrane (PEM) electrolyzers, which function in acid, can operate at current densities that are a factor of three to four higher than alkaline electrolyzers and they are more stable towards load-cycling and shutdowns. ${ }^{7}$ Today, PEM electrolyzers typically use platinum loadings of $0.5-1.0 \mathrm{mg} \mathrm{cm}^{-2}$ but operation at lower loadings has been demonstrated; for example, $3 \mathrm{M}^{\prime}$ 's nano-structured thin film (NSTF) with $0.25 \mathrm{mg} \mathrm{st}_{\mathrm{Pm}}^{-2}$ at the cathode (and $0.25 \mathrm{mg}_{\mathrm{Ir}} \mathrm{cm}^{-2}$ at the anode) maintained stable operation at $2.0 \mathrm{~A} \mathrm{~cm}^{-2}$ for $5,000 \mathrm{~h}^{8}{ }^{8}$ This corresponds to $\sim 100$ tonne platinum for $1 \mathrm{TW}_{\mathrm{H}_{2}}$, close to $60 \%$ of the current annual production. For both PEC and PEM electrolyzers it may thus be possible to scale platinum-based cathodes to the TW-level.

For the OER in acid, the best catalyst, considering both activity and stability, is $1 \mathrm{rO}_{2} \cdot{ }^{9}$ Although $\mathrm{RuO}_{2}$ is more active (Figure $2 \mathrm{~A}$ ) and is often used together with $\mathrm{IrO}_{2}$, it is also less stable. However, even iridium oxide is not entirely stable under OER. ${ }^{10}$ Unfortunately, iridium is an even more critical element than platinum. It is only produced in small amounts ( $<10$ tonne/year) as a byproduct of the production of the other platinum group metals ${ }^{3}$ and its price has more than doubled over the last decade ${ }^{11}$. Recently, a very large current density of $100 \mathrm{~A} \mathrm{glr}^{-1}$ (roughly 6.5 $\mathrm{mA} \mathrm{cm}{ }^{-2}$ ) at $1.51 \mathrm{~V}$ was claimed for a rotating disk setup, ${ }^{12}$ a technique where the electrode is being rotated to limit the diffusion layer thickness. This corresponds to an overpotential of $~ 270$ $\mathrm{mV}$ and an efficiency of $82 \%$, but would require $~ 8100$ tonne iridium for $1 \mathrm{TW}_{\mathrm{H}_{2}}$. It was further shown that $2 \mathrm{~A} \mathrm{~cm}^{-2}$ at a cell potential of $1.85 \mathrm{~V}$ with a catalyst loading of $1 \mathrm{mg}_{\mathrm{Ir}} \mathrm{cm}^{-2}$ could be obtained in a PEM electrolyzer. This corresponds to $2000 \mathrm{~A} \mathrm{gIr}^{-1}$ at $\sim 64 \%$ efficiency, and would require 400 tonne iridium to produce $1 \mathrm{TW}_{\mathrm{H}_{2}}$. As discussed earlier for platinum ${ }^{5}$, these numbers demonstrate how we can trade overpotential for loading, and suggest that we would still need roughly 40 years of iridium production to achieve $1 \mathrm{TW}_{\mathrm{H}_{2}}$. Although better PEM electrolyzer performance has been obtained, (for example, the 3M NSTF catalyst achieves a current density of $2.0 \mathrm{~A} \mathrm{~cm}^{-2}$ at $1.85 \mathrm{~V}$, using only $0.25 \mathrm{mg} \mathrm{cm}^{-2}$ iridium at the anode) $)^{8}$, even highly optimized systems would still currently require at least 100 tonne iridium for $1 \mathrm{TW}_{\mathrm{H}_{2}}$. Evidently, compared to platinum, iridium is a true bottleneck for production of hydrogen on the terawatt scale. While partly due to iridium's rarity, this difference is also a testament to the exceptionally good HER activity of platinum and the limitations that the scaling relations, discussed below, impose on current OER catalysts, including iridium. A comparison between HER activity of platinum and the OER activity for a state-of-the-art iridium-based catalyst ${ }^{12}$ (Figure $1 \mathrm{~A}$ ) underscores this, demonstrating 3-4 orders of magnitude difference.

\section{Alternative catalysts for HER and OER}

Although platinum-based cathodes potentially can be scaled to the TW-level, platinum has many other technological - and perhaps more critical - uses ${ }^{13}$; it would thus be desirable if earthabundant catalysts could be developed that could outcompete, and replace, platinum. In the last decade, first sulfides ${ }^{14,15}$ and later phosphides ${ }^{16-18}$ have been found to be good catalysts for HER, and now overpotentials for a specific current per geometric area (ie current divided by the projected area of the electrode) are reported to be so close to platinum that further improvements seem needless. ${ }^{1}$

However, in reality it only appears so because many catalytic sites - usually achieved through using high loadings of catalysts or by increasing their surface area - are employed to compensate 
for poorer intrinsic activity. Increasing the geometric activity by greatly increasing the loading may in the end not be a viable route, since in many cases this would be even more costly than using platinum. ${ }^{5}$ Hence, looking solely at the geometric activity of a catalyst gives a false picture of whether it can be used at large scale.

To evaluate and compare catalytic activity and the potential for scalability, it is therefore more instructive to look at the activity per unit mass or, even better, the turn over frequency (TOF), which quantifies how many molecules (in the case of water splitting: $\mathrm{H}_{2}$ and $\mathrm{O}_{2}$ ) are evolved per second per site at a specific overpotential. This is the relevant scientific activity metric for comparing intrinsic activity. The technological aspect is naturally how you can get as many of those in a limited space without running into transport problems. It is currently not possible to establish a consistent overview of published data based on TOF since the number of active sites is often unknown or unreported. However, a lower limit on the TOF can be established by assuming that all atoms present are active, or as mentioned earlier, the activity normalized by the mass of catalyst can be compared. This method demonstrates that while phosphide-based catalysts are approaching the activity of platinum-based catalysts measured per geometric area for acidic HER (Figure $1 \mathrm{~A}$ ), it is at the expense of a high catalyst loading that severely lowers the mass activity. Similar observations can be made for activity under alkaline conditions (Figure 1B).

The search for earth-abundant alternatives for platinum is important, but we suggest that research efforts should be directed towards increasing their intrinsic activity and not just the number of available sites. Whether non-precious catalysts can be tailored to exhibit true platinumlike activity on a TOF basis and to what degree they are stable remains an open question. ${ }^{19}$

While there is still work to do for HER in both acid and alkaline conditions, it is the OER that leads to the largest overpotential, even when using precious metals, and thus where there is the biggest potential for gains in efficiency by finding better catalysts. ${ }^{1}$ The OER is a four-electron process involving multiple intermediates (such as $\mathrm{OOH}^{*}, \mathrm{OH}^{*}, \mathrm{O}^{*}$ ), of which the binding energies are strongly correlated and cannot be decoupled easily due to scaling relations. ${ }^{1}$ Scaling relations provide linear relationships between the binding energies of the intermediates and hence cannot be optimized independently, posing a fundamental limitation to the catalytic efficiency. This has stagnated the development of more active OER catalysts for decades leading to only incremental improvements. ${ }^{1}$ Research is today focused on breaking those scaling relations to obtain lower overpotentials.

Similar to HER, OER activities are often reported as the overpotential required to achieve a current density of $10 \mathrm{~mA} \mathrm{~cm}^{-2}$, since this corresponds to harvesting slightly above $10 \%$ of the sunlight in a $\mathrm{PEC}$ device. As pointed out above, it would be highly desirable if a TOF could be estimated but often it is even more difficult to isolate the actual number of active sites on these typical oxide catalysts than it is for HER. In Figure 2A, we therefore again plot a comparison of mass and geometric activity for OER in acidic media; note that all catalysts are based on ruthenium- or iridium-based since only they display sufficient stability. It is evident that on both metrics, the OER catalysts are not as active as the HER catalysts in Figure $1 \mathrm{~A}$. 
Despite the drawbacks compared to PEM electrolyzers, alkaline electrolyzers have the major advantage that some of the best catalysts for OER under alkaline conditions are based on abundant elements such as iron and nickel. These catalysts have been used for decades, with an overpotential of $\sim 300 \mathrm{mV}$ for $10 \mathrm{~mA} \mathrm{~cm}{ }^{-2}$ at low loading $\left(1-100 \mu \mathrm{g} \mathrm{cm}^{-2}\right)^{20}$, but better catalysts are still being developed (Figure 2B). Similar to HER, many studies have focused on increasing the geometric activity by greatly increasing the loading, as is evident from Figure $2 \mathrm{~B}$, which can lead to several pitfalls as pointed out below.

\section{Pitfalls for erroneous data}

The competition for finding better HER and OER catalysts has led to an emphasis on claiming activity records. Unfortunately, several pitfalls for erroneous data exist that must be avoided, not least when claiming records on activity.

Potential scale calibration: Although it seems trivial, one must first establish the reversible hydrogen electrode (RHE) scale by employing a platinum electrode to measure the reversible potential in the particular setup used, ensuring that the electrolyte is saturated with hydrogen. The electrolyte and cell should be rigorously clean to avoid poisoning of the platinum electrode.

Counter electrode: For HER, the use of a platinum counter electrode should be entirely avoided, since even a trace amount of platinum anodically corroding out in the electrolyte can re-deposit on the working electrode and enhance the apparent activity.

Product detection: The actual amount of gas evolved should be measured instead of only reporting the associated current; this is especially critical for OER. Very high geometric activity has been reported for materials with a large surface area and mass loading with components that can easily be oxidized, e.g. carbon, sulfur or phosphorus (see Figure 2B). ${ }^{21,22}$ This could result in a misleading current that has nothing to do with oxygen evolution, but rather a change of the catalyst oxidation state. Thus, if a record should be claimed, the amount of oxygen evolved must be measured at the record potential. For example, if $10 \mathrm{~mA}$ of true OER current can be established, it will only take 1 hour to produce $2 \mathrm{ml}$ of $\mathrm{O}_{2}$, which is easily measurable - but rarely done.

State-of-the-art: The obtained activity should be compared with the state-of-the-art. Homemade, subpar platinum electrodes are too often used instead of state-of-the-art literature values. As shown in Figure 2B, for alkaline OER the state-of-the-art catalyst is not platinum, ruthenium, nor iridium and comparison to these precious metals is thus irrelevant.

Stability: For both HER and OER, stability tests are often performed on electrodes with a large catalyst loading and tested at constant current (chronopotentiometric) over a few hours; if no change in the potential is measured, it is claimed stable. Unfortunately, this method is insufficient and should only be used as a preliminary test. A corroding thick film could even improve over time as it gets rougher (due to a higher electrochemical active surface area) and then entirely fail when all the material has corroded away. For more rigorous stability testing one should either work at very low catalyst loadings (sub-monolayers) or - better yet - measure if there are any corrosion 
products in the electrolyte with an ICP-MS analysis, see for example ref. ${ }^{19}$. This is an extremely sensitive technique and one can easily predict stabilities issues due to corrosion over years ${ }^{23}$.

Turn over frequency: It would be extremely useful and more scientifically interesting if TOF could be established more securely, but it remains a challenge to identify the number of active sites on many catalysts. Figure $2 \mathrm{C}$ shows how the activity ranking for metal oxides can change substantially when these are ordered by TOF rather than geometric current density. It should however be remarked that all catalysts displayed here are fundamentally limited by the scaling relations, which has hampered further progress; for example, NiFe is a well-known catalyst and the overpotential is still substantial. ${ }^{24}$

\section{Outlook}

It is extremely important that good and trustworthy data are presented when claiming records - in particular for OER - as, to our knowledge, there are basically no examples where scaling relations have been broken. An overpotential defined by a DFT-based microkinetic model for a specific TOF resulting in a certain current density, e.g. $10 \mathrm{~mA} \mathrm{~cm}^{-2}$, and obtained for a planar surface of active sites provides a predicted reference for intrinsic activity. ${ }^{25,26}$ If the number of sites per geometric area is substantially increased, the overpotential can easily be reduced far below this predicted activity for a planar surface. However, increasing the loading or surface area does not equate to breaking scaling relations, illustrating the importance of knowing the activity per site. A radically increased TOF at a given overpotential (or decrease in overpotential for a given TOF), as compared to DFT-based values bound by scaling relations, will be the best indication of that scaling relations have been broken, or that the entire concept is simply wrong. Both outcomes are interesting from a scientific point of view and should be confirmed by detailed structural and mechanistic investigations.

In terms of catalyst development, it is clear that the higher gain is for the OER process where substantial overpotentials still prevail and there is an issue of scalability for the acidic reaction. While scaling platinum-based catalysts to the TW-level may not be prohibitive for HER, it is simply not possible to scale iridium-based catalyst to the TW-level for OER. In general, we therefore urgently need to discover new OER catalysts both to lower the overpotential and to operate in acidic conditions if we are to use acidic electrolysis on the TW scale.

\section{Data availability}

The source data for figures are available in Supplementary Data 1.

\section{Competing Interests}

The authors declare no competing interests. 

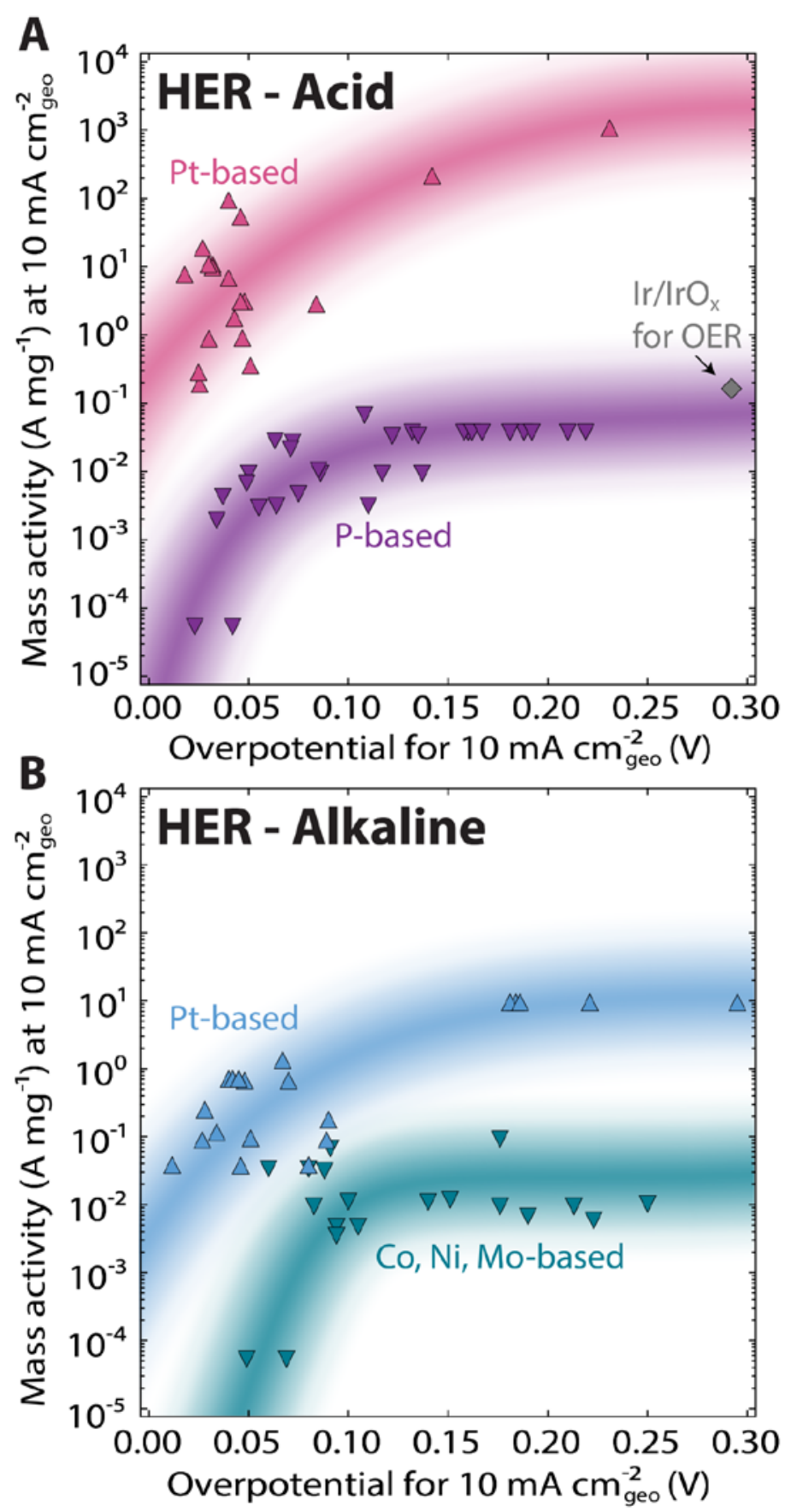

Figure 1. Comparison of mass activity and overpotential for hydrogen evolution catalysts. (A) Mass activity measured at $10 \mathrm{~mA} \mathrm{~cm}^{-2}$ and the overpotential for $10 \mathrm{~mA} \mathrm{~cm}^{-2}$ (based on geometric area) for platinum and non-noble transition metal phosphide-based catalysts for acidic HER. A data point for $\mathrm{Ir} / \mathrm{IrO}_{x}$ for OER is shown in grey for comparison. (B) The same parameters for platinum, $\mathrm{Co}, \mathrm{Ni}$ and Mo-based catalysts for alkaline HER. Shadings are guide to the eye. For data and references see Supplementary Data 1. Note that the reported activities of platinum are nearly always transport limited and thus likely underestimated. This is especially true when high loadings or a planar platinum electrode is used. Attempts to largely eliminate transport problems have resulted in estimated currents of $250 \mathrm{~A} \mathrm{mg}^{-1}$ for $2 \mathrm{~nm}$ platinum particles at $10 \mathrm{mV}$ overpotential for both HER and HOR. ${ }^{27}$ 

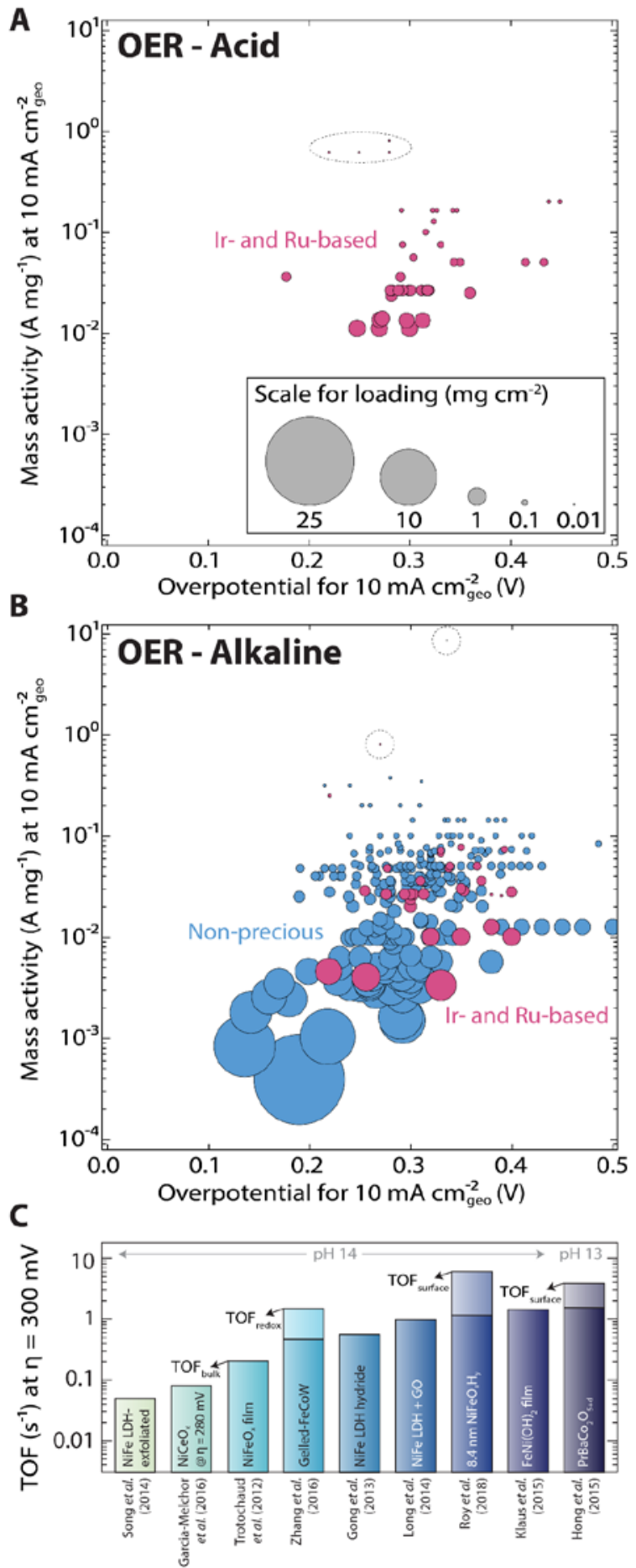

Figure $\mathbf{2}$ Comparison of mass activity and overpotential for oxygen evolution catalysts (A) Comparison of mass activity measured at $10 \mathrm{~mA} \mathrm{~cm}^{-2}$ and the overpotential for $10 \mathrm{~mA} \mathrm{~cm}^{-2}$ (based on geometric area) for (A) ruthenium- and iridium-based catalysts for acidic OER. and (B) The same parameters for ruthenium- and iridium-based and non-precious-metal based catalysts for alkaline OER. The areas of the data points are proportional to the mass loading per $\mathrm{cm}^{2}$ - see scale in (A). For clarity, dashed circles highlight points with extremly low mass loading. For data and references see Supplementary Data 1. (C) TOF of state-of-the-art OER catalysts in alkaline measured at $300 \mathrm{mV}$ overpotential. TOF bulk $_{\text {bssumes all atoms are active, TOF }}$ surface

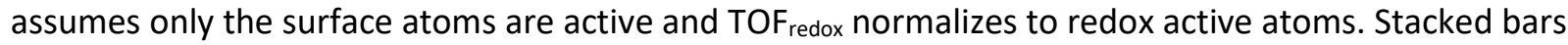
display both TOF $F_{\text {bulk }}$ value and a larger either TOF surface or TOF redox value. Adapted from ref. ${ }^{24}$ 


\section{REFERENCES:}

1. Seh, Z. W. et al. Science (80-. ). 355, 146 (2017).

2. $\quad$ Ardo, S. et al. Energy Environ. Sci. 11, 2768-2783 (2018).

3. Vesborg, P. C. K. \& Jaramillo, T. F. Rsc Adv. 2, 7933-7947 (2012).

4. IEA (2018), World Energy Outlook 2018, IEA, Paris. (2018). doi:10.1787/weo-2018-en

5. Kemppainen, E. et al. Energy Environ. Sci. 8, 2991-2999 (2015).

6. Zhang, J. et al. Nat. Catal. 1, 985-992 (2018).

7. Ayers, K. E. et al. ECS Trans. 33, 3-15 (2010).

8. Lewinski, K. A., van der Vliet, D. F. \& Luopa, S. M. ECS Trans. 69, 893-917 (2015).

9. Seitz, L. C. et al. Science (80-. ). 353, 1011-1014 (2016).

10. Kasian, O., Grote, J. P., Geiger, S., Cherevko, S. \& Mayrhofer, K. J. J. Angew. Chemie - Int. Ed. 57, 2488-2491 (2018).

11. Laursen, A. B., Sehested, J., Chorkendorff, I. \& Vesborg, P. C. K. Chinese J. Catal. 39, (2018).

12. Lettenmeier, P. et al. Chem. Sci. 9, 3570-3579 (2018).

13. Cowley, A. ISSN 0268-7305 (2013).

14. Hinnemann, B. et al. J. Am. Chem. Soc. 127, (2005).

15. Jaramillo, T. F. et al. Science 317, 100-102 (2007).

16. Popczun, E. J. et al. J. Am. Chem. Soc. 135, 9267-9270 (2013).

17. Kibsgaard, J. et al. Energy Environ. Sci. 8, 3022-3029 (2015).

18. Callejas, J. F., Read, C. G., Roske, C. W., Lewis, N. S. \& Schaak, R. E. Chem. Mater. 28, 60176044 (2016).

19. Ledendecker, M. et al. Angew. Chemie Int. Ed. 56, 9767-9771 (2017).

20. Dionigi, F. \& Strasser, P. Adv. Energy Mater. 6, 1600621 (2016).

21. Zhao, S. et al. Nat. Energy 1, 16184 (2016).

22. You, B. \& Sun, Y. Adv. Energy Mater. 6, 1502333 (2016).

23. Frydendal, R. et al. Chemelectrochem 1, 2075-2081 (2014).

24. Roy, C. et al. Nat. Catal. 1, 820-829 (2018).

25. Hansen, H. A., Viswanathan, V. \& Nørskov, J. K. J. Phys. Chem. C 118, 6706-6718 (2014).

26. Ping, Y., Nielsen, R. J. \& Goddard, W. A. J. Am. Chem. Soc. 139, 149-155 (2017).

27. Zalitis, C. M., Kucernak, A. R., Sharman, J. \& Wright, E. J. Mater. Chem. A 5, 23328-23338 (2017). 